\title{
Análisis de la Gestión de la Oferta Educativa del Tercer Ciclo de la Educación General Básica Abierta en la Dirección Regional de Educación de Occidente
}

\author{
Management Analysis of Educational Offer in Third Cycle of \\ Distance Education of Basic General Education in the Dirección \\ Regional de Educación de Occidente (West Regional Education \\ Bureau)
}

Recibido 21 enero 2012 • Aceptado 14 mayo 2012 • Corregido 30 mayo 2012

\author{
Alfaro Céspedes Mildred ${ }^{1}$ \\ Ministerio de Educación Pública, \\ San José, Costa Rica \\ mil-mac@hotmail.com \\ Arce Barrantes Margoth ${ }^{2}$ \\ Ministerio de Educación Pública, \\ Naranjo, Costa Rica \\ gori38@gmail.com \\ Gatjens Esquivel Marianela ${ }^{3}$ \\ Ministerio de Educación Pública, \\ San Ramón, Costa Rica \\ nelarisitas@hotmail.com
}

\footnotetext{
1 Licenciada en Ciencias de la Educación con énfasis en Administración de la Educación de la Universidad de Costa Rica (UCR). Bachiller en Enseñanza de los Estudios Sociales de la Universidad de Costa Rica (UCR) y es Licenciada en la Enseñanza de los Estudios Sociales de la Universidad San Isidro Labrador. Actualmente labora como Auxiliar administrativo en la Educación Secundaria del Sistema educativo Público.

2 Licenciada en Ciencias de la Educación con énfasis en Administración de la Educación de la Universidad de Costa Rica (UCR). Bachiller en Ciencias de la Educación con énfasis en Educación Preescolar de la Universidad de Costa Rica (UCR). Actualmente labora como docente de Educación Primaria del Ministerio de Educación Pública.

3 Licenciado en Ciencias de la Educación con énfasis en Administración de la Educación de la Universidad de Costa Rica (UCR). Actualmente labora como profesor de los Programas de Educación Abierta para Jóvenes y Adultos en la Universidad de Costa Rica y como profesor del Colegio Nacional virtual Marco Tulio Salazar, sede Tejar de El Guarco, Cartago.
} 
Murillo Vega María Teresa ${ }^{4}$ Ministerio de Educación Pública, Heredia, Costa Rica maritemv.12@gmail.com

\section{Quintanilla Moran José Reynold ${ }^{5}$}

Ministerio de Educación Pública,

Cartago, Costa Rica quinta5755@hotmail.com

Vásquez Pacheco Siany ${ }^{6}$ Ministerio de Educación Pública,

San José, Costa Rica sianyvasquez@hotmail.com

Resumen. Los autores presentan en este artículo los resultados de una investigación que tuvo como objetivo general analizar la gestión de la oferta educativa del III Ciclo de la Educación General Básica Abierta para Jóvenes y Adultos en la Dirección Regional de Educación de Occidente. Es un estudio exploratorio y descriptivo, el cual utilizó técnicas de investigación cualitativas. Para la recolección de la información se emplearon instrumentos como cuestionarios y entrevistas que se aplicaron a los actores que intervienen directamente en esta modalidad de educación abierta. El análisis de datos se presenta según las categorías de análisis que fundamentaron el estudio: Sistema de coordinación desde la gestión, planificación de las acciones en las sedes y los proyectos, la organización de la modalidad en las sedes y proyectos, ejecución de las acciones administrativas y la evaluación. Entre los principales hallazgos se hacen notar: la deficiente comunicación de los lineamientos a través de los niveles macro, medio y micro dentro de las diferentes instancias, ausencia de espacios de involucramiento para la comunidad educativa, inadecuada previsión y distribución de los recursos financieros, humanos y didácticos de la modalidad educativa, currículo poco adaptado a las necesidades de la población meta y una evaluación que desde la gestión no produce insumos para mejorar la modalidad.

4 Licenciada en Ciencias de la Educación con énfasis en Administración de la Educación de la Universidad de Costa Rica (UCR). Bachiller en Enseñanza de los Estudios Sociales de la Universidad de Costa Rica (UCR) , cursa una Licenciatura en la Enseñanza de los Estudios Sociales en la Universidad de Costa Rica (UCR). Actualmente labora como Asistente de Dirección de Educación Secundaria del Sistema Educativo Público.

5 Licenciado en Ciencias de la Educación con énfasis en Administración de la Educación de la Universidad de Costa Rica (UCR). Licenciado en Ciencias de la Educación con énfasis en Administración de la Educación de la Universidad de Costa Rica (UCR). Bachiller en Enseñanza de los Estudios Sociales de la Universidad de Costa Rica (UCR), cursa una Licenciatura en la Enseñanza de los Estudios Sociales en la Universidad de Costa Rica (UCR). Actualmente labora como Asistente de Dirección de Educación Secundaria del Sistema Educativo Público

6 Licenciada en Ciencias de la Educación con énfasis en Administración de la Educación de la Universidad de Costa Rica (UCR). Licenciada en Educación General Básica con énfasis en I y II Ciclo de la Universidad Estatal a Distancia (UNED). También es Bachiller en Educación General Básica con énfasis en I y II Ciclo de la Universidad Estatal a Distancia (UNED). Actualmente se desempeña como Asistente de Supervisión de Centros Educativos del Ministerio de Educación Pública. 
Palabras claves. Administración de la educación, gestión de la educación, educación abierta, andragogía, sistema educativo costarricense.

\begin{abstract}
The authors present, in this article, results of a research analyzing the overall management of learning distance educational offers of Cycle III General Basic Education for Youth and Adults in Dirección Regional de Educación de Occidente (West Regional Education Bureau). This is an exploratory and descriptive research using qualitative techniques. In order to collect information, questionnaires and interviews were applied to the actors directly involved in this type of learning distance education. Data analysis is presented in analysis categories that support this study: system coordination from management, action planning at projects locations, the organization of learning distance projects locations, implementation of administrative actions and evaluation. Among the key findings are noted: poor communication of the guidelines towards different levels from the macro, meso and micro educational levels, lack of involvement of the educational community, inadequate provision and distribution of financial, human and teaching resources, unsuitable curriculum for the needs of target population, and an inadequate assessment which does not produce inputs to improve this kind of education.
\end{abstract}

Keywords. Educational administration, educational management, distance education, distance learning, andragogy, Costa Rican educational system.

\title{
Introducción
}

Costa Rica, se ha preocupado por dotar de una educación de calidad a sus habitantes universalizando la educación primaria y ampliando la cobertura de la educación secundaria, permitiendo así el fortalecimiento de una clase media que ha propiciado una movilidad social; no obstante, a pesar del esfuerzo aún quedan sectores de la sociedad excluidos del sistema educativo formal, por lo tanto grupos de personas adultas y jóvenes mayores de 15 años, optan por ingresar a la oferta de Educación de personas jóvenes y adultas (EDJA), la cual tiene por misión brindar un servicio de oferta educativa a todas aquellas personas que por diversas razones no han concluido la educación formal en sus distintos niveles. En tanto, la EDJA visualiza una alternativa de oportunidades educativas flexibles, innovadoras y novedosas de acuerdo con las necesidades, intereses y demandas de la población beneficiaria; con la intención de disminuir las fracturas sociales, que apuntan hacia una mayor profundización de las diferencias (MEP, 2010).

Los fines planteados para la Educación Abierta para Jóvenes y Adultos en el sistema educativo costarricense son, según el Ministerio de Educación Pública (MEP, 2010): 
- El desarrollo armónico y pleno de la personalidad.

- La formación de ciudadanos amantes de su patria, con su propio sentido de la responsabilidad y de respeto a la dignidad.

- La formación de ciudadanos aptos para construir una democracia que concilie los intereses del individuo con los de la comunidad.

- La conservación, fomento y difusión de la cultura.

- El logro de una persona capaz de organizarse en las funciones de las transformaciones necesarias para un mundo de paz y justicia.

- La capacidad de construir una sociedad en la que se concilie los intereses de esta con los del individuo.

- El aprovechamiento del desarrollo universal del conocimiento, así como el avance tecnológico y científico, en función de la formación integral de la persona y la sociedad. (p. 12-13)

La modalidad de Educación Abierta que el sistema de educación ofrece, como otros sistemas de educación formal, involucra procesos administrativos, los cuales presentan ciertas complejidades en la gestión, visto desde la coordinación, la planificación, la organización, la ejecución y la evaluación. Al respecto, la administración de la educación no debe estar aislada, sino inmersa dentro de los retos que ofrece el contexto en el cual esta modalidad se desenvuelve, es así, que surge la necesidad desde este campo disciplinario de analizar la gestión de la modalidad de la oferta educativa del tercer ciclo de la educación general básica abierta.

\section{Fundamentación teórica}

\section{Administración.}

En el mundo actual la administración debe tener un carácter dinámico y evolutivo, para adaptarse a los constantes cambios de la sociedad, en este sentido definir administración es complejo, para ello se adopta a la definición propuesta por Terry (1980, p.101) que define la administración como "un proceso distintivo compuesto por planeación, organización, ejecución 
y control, que se ejecuta para determinar y satisfacer los objetivos establecidos mediante el uso de gente y otros recursos". Como se menciona, la administración debe satisfacer los objetivos propuestos; para esto necesita de diferentes funciones y mantener un control de las acciones que se desarrollan en cierto contexto; sin dejar de lado los recursos con los que cuenta el administrador para llevar a cabo el proceso de gestión.

Según Chiavenato (2006), en un mundo complejo y dinámico, la administración se ha convertido en una de las áreas más importantes de la actividad humana:

Vivimos en una civilización donde predominan las organizaciones y donde el esfuerzo cooperativo del hombre es la base fundamental de la sociedad. La tarea básica de la administración es hacer las cosas por medio de las personas de manera eficaz y eficiente. En las organizaciones la eficiencia y la eficacia con que las personas trabajan en conjunto para conseguir objetivos comunes dependen directamente de la capacidad de quienes ejercen la función administrativa (p. 10).

El reto ha sido pasar de la teoría a la práctica y hacer que los postulados propuestos por las diferentes concepciones del término administración se apliquen a la educación. En este sentido la administración de la educación debe regirse por principios similares y utilizar estos parámetros para dirigirse al cumplimiento de sus metas; es por esto que la administración de la educación se vuelve un instrumento necesario para conocer más sobre la gestión que se debe llevar a cabo en la modalidad de educación abierta.

\section{Administración de la Educación.}

Integrar la administración en los centros educativos requiere tomar en cuenta diversos factores que a corto o largo plazo beneficiarán la educación en todo su ámbito, lo cual le permitirá responder a lo que la sociedad exige en esta época.

Se sabe que para el manejo adecuado de un centro educativo es necesario que su administración esté ligada a las acciones sociales que se realizan en las diferentes comunidades; ya que en gran medida muchos de los problemas que los administradores deben 


\section{Gi: Eestión:}

Revista Cientifica Digital ISSN:2215-2288

[Número public ado el 30 de J unio del 2012]
URL:http :/ / revista dig ita l.ea e.fcs.ucr.ac.cr/

enfrentar se refieren a dificultades que nacen en la sociedad, tales como desempleo, drogas, delincuencia, entre otros.

Costa Rica se preocupa por la educación de sus habitantes, por lo que esta pasa a ser una empresa de gran importancia a nivel nacional, de la cual se puede llegar a obtener grandes resultados; por dicha razón se hace necesaria una buena administración de los centros educativos.

Así mismo, Chavarría y Araya (1984) establecen un concepto de administración muy relacionado con el contexto actual en el ámbito de la educación, donde explica:

(...) es un proceso integrado por medio del cual se traza una política educativa encaminada al logro de los fines claramente determinados con base en las necesidades del país y en las aspiraciones del grupo, tomando en cuenta las características de la época.

Es un sistema en que cada elemento es parte vital y guarda estrecha relación con los demás. Es un proceso en que deben cumplirse, entre otras, las siguientes etapas: investigación, definición del problema, planeamiento, organización, acción y ejecución, evaluación y rectificación.

La administración ha de ser un instrumento dinámico, capaz de convertir a una organización en una actividad efectiva, concebida como un sistema de vasos comunicantes y que la oficina central sea órgano de servicio en donde coordinen y se unifiquen las diferentes labores. (p. 46)

Es así, como la administración tiene un papel primordial en la planificación, organización, coordinación, control del proceso educativo y cuyos fines sean el desarrollo del currículo, lo pedagógico y lo humano factores que afectan la calidad de la educación, en otras palabras debe buscar los contenidos educativos y garantizar a través de los métodos idóneos un procedimiento eficaz de enseñanza-aprendizaje, en función de determinados objetivos de cantidad y calidad, en un período definido.

Toda institución educativa debe fijar sus metas y objetivos, tomando en cuenta tanto los factores internos como externos que intervienen en el proceso administrativo; favoreciendo como se refirió la calidad del desarrollo de enseñanza y aprendizaje. 
Para comprender cómo interacciona la administración en la educación, conviene dar una revisión de las características de la Administración Educativa. En este sentido Chavarría y Araya (1984) definen que los caracteres que debe reunir la administración educativa son:

-Dinámica, de modo que permita decisiones rápidas, flexibles y eficaces, asegurando la optimización de los recursos humanos, financieros y técnicos que sean necesarios. -Innovadora, incluyendo nuevos procedimientos administrativos, nuevas disposiciones, cambios estructurales y nuevos métodos educativos.

-Previsora, a través del planeamiento y los controles permanentes establecidos según técnicas modernas de la administración.

-Estable, constituida por profesionales de alto nivel, no sujetos en la medida de lo posible, a los cambios de la naturaleza gubernamental.

-Participativa, con la intervención de los distintos elementos que forman parte del sistema educativo, es decir, alumnos, docentes, partes, grupos sociales, y comunidad, de tal modo que sea posible una determinación más racional de las políticas y objetivos de la educación (...) (p. 6).

El campo de la administración es muy amplio, convirtiéndose la administración de la educación en un ente integrador que consciente de sus objetivos, coordina y orienta la actividad de los otros elementos hacia el logro de los mismos objetivos. Resumiendo, puede decirse que la función de la administración de la educación es general y dinamizante de la acción del sistema.

\section{Gestión de la educación.}

El mundo actual, globalizado y dinámico; demanda que los sistemas educativos desarrollen estrategias de gestión que respondan a las demandas de una sociedad cada vez más compleja; reconocer los cambios coyunturales es por tanto, un paso necesario para todo administrador. La tarea de gestionar es complicada, no obstante, debe tener presente que debe coadyuvar al éxito y la calidad de las instituciones con las estrategias asertivas necesarias que permitan brindar un servicio de calidad a la sociedad; como bien apunta Esquivel (2009, p.42) 
[Número public ado el 30 de J unio del 2012]

"el administrador de la educación se convierte en un gestor que debe desarrollar estrategias de calidad en la institución".

A partir de lo anterior, se reafirma que el proceso educativo precisa en la actualidad una gestión que favorezca el éxito, un curso dinámico que debe enlazar las contrariedades de la sociedad con el acto educativo y a su vez buscar los mecanismos adecuados para ofrecer soluciones alternativas que mejoren el fenómeno de estudio.

En términos generales se puede afirmar que la práctica de la gestión va mucho más allá de la mera ejecución de instrucciones que vienen del centro; como sucedía con la administración. Al aplicarla las personas tienen responsabilidades de conducción, tienen que planificar y ejecutar el plan adecuándolo a su realidad (operaciones de ajuste), es claro que el concepto de gestión connota tanto las acciones de planificar como de administrar.

El término gestión educativa es relativamente reciente; como señala Casassus, (2000):

La gestión educativa data de los años sesenta en Estados Unidos, de los años setenta en el Reino Unido y de los años ochenta en América Latina. Es por lo tanto, una disciplina de desarrollo muy reciente. Por ello, tiene un bajo nivel de especificidad y de estructuración. (p. 2)

Lo anterior refleja el hecho que exista una amplia gama de literatura referida al término gestión a nivel empresarial de carácter técnico e instrumental asociado al direccionamiento y a los resultados, mas no así, al término gestión educativa.

De acuerdo con lo expresado con Casassus (2000):

La gestión educativa busca aplicar los principios generales de la gestión al campo específico de la educación. El objeto de la disciplina es el estudio de la organización del trabajo en el campo de la educación. Por tanto está determinada por el desarrollo de las teorías generales de la gestión y de la educación. (p. 2) 
Queda claro que el término gestión educativa deriva del término gestión vinculada al ámbito empresarial, por lo que no es de extrañar que haya permeado en el ambiente escolar una gestión que parta de los supuestos de reducción de costos y beneficios; aspectos que se convierten en un dilema si los trasladamos al ámbito educativo, es pues, evidente que las demandas del mercado influyen cada vez más la dinámica educativa legitimando el modelo hegemónico neoliberal.

La gestión educativa es concebida como un proceso mediante al cual se motiva a participar responsablemente a los principales implicados en la labor educativa (bajo la supervisión del director) para tomar las decisiones que incidan positivamente en la calidad de los servicios así Castillero (2003, p.10), visualiza la gestión educativa como "el conjunto de actuaciones que se desarrollan en el centro como expresión del gobierno institucional, bajo la conducción y responsabilidad del director para definir sus objetivos y diseñar la naturaleza y desarrollo para alcanzarlos".

La gestión educativa debe ser vista como un conjunto de procesos teóricos y prácticos integrados horizontal y verticalmente dentro del sistema educativo para cumplir los mandatos formales, en donde la gestión debe ser entendida como una nueva forma de comprender y conducir la organización escolar.

La gestión en Costa Rica debe velar por una educación de calidad, gestionada en primera instancia por el Estado -el cual debe asumir su papel como garante del bien común, y gestionar de manera amplia el derecho a la educación como bien público-. Esta intervención se debe hacer con dos tipos de acciones: por un lado dirigiendo cambios profundos en el diseño del modelo de enseñanza en la organización y gestión del sistema educativo por el otro. Una adecuada gestión facilita que la organización educativa logre alcanzar los objetivos y metas propuestas, así mismo, permite que todos los responsables de la organización valoren el logro alcanzado en nuevos propósitos educativos, para que estos sean ejemplo ante la comunidad educativa regional y nacional.

Reconociendo la importancia de la aplicación del concepto de gestión a la praxis escolar, se debe visualizar este como un ciclo. Por las consideraciones anteriores, se establece que el ciclo de la gestión, para efectos de esta investigación, se estructura de la siguiente forma: coordinación, planificación organización, ejecución y evaluación. 
Se debe aclarar que se coloca la coordinación como primera etapa puesto que es el punto medular del tema en estudio, debido a que se pretende determinar la gestión que se desarrolla en la oferta educativa donde existe coordinación y donde solo se desarrolla proyectos.

\section{Coordinación.}

Los centros educativos para realizar un adecuado trabajo, no dependen únicamente de la buena labor que desempeñen los educadores; se hace necesaria una gestión que brinde a la institución los medios por las cuales enfrentarse a las circunstancias diarias.

Como parte de esta gestión el administrador (a) o director (a) en este caso, debe poseer varias características que le permitan desempeñarse de la mejor manera. Entre estas, se encuentra la de coordinador y en relación con la misma Guadamuz $(1979$, p.42) indica que "EI coordinador (...) es el responsable de integrar los esfuerzos aislados e independientes de los diversos sectores o de los diversos especialistas". Como tal, es el que se encarga de llevar a cabo las acciones administrativas, curriculares, financiera y de infraestructura, que son necesarias para un adecuado proceso de gestión.

Al encontrarse al mando de un grupo de personas, el coordinador debe buscar la forma de unificar criterios de los diferentes actores del proceso de enseñanza y aprendizaje; tratando de mantener un equipo de trabajo que labore de acuerdo con las necesidades del centro educativo.

Koontz, Weihrich y Cannice (2008, p.476) consideran que: "un equipo puede definirse como un pequeño número de personas con habilidades complementarias y comprometidas con un propósito común, serie de metas de desempeño y enfoque de los que son mutuamente responsables". En este caso mantener un adecuado equipo de trabajo (Director, Secretario o Asistente Administrativo, Comité Coordinador, Junta Administrativa, Asociación de Padres de Familia, gobierno estudiantil) y unificar esfuerzos en las organizaciones educativas permitirá desarrollar buenos proyectos que mejoren su desempeño, para esto se hace necesario que se ejecuten las acciones pertinentes para llevar a cabo una buena coordinación.

En el área curricular, desde la coordinación, el administrador debe tomar muy en cuenta cómo desarrollan el currículo los docentes de la institución, de acuerdo con esto y como lo citan 
Bolaños y Molina (2008, p.23): "Currículo son todas las experiencias, actividades, materiales, métodos de enseñanza y otros medios empleados por el profesor o tenidos en cuenta por él en el sentido de alcanzar los fines de la Educación"; por lo tanto, mediante la coordinación se puede determinar la aplicabilidad del currículo en el sistema educativo.

La gestión no depende únicamente de las acciones del director(a), sino que abarca una serie de personas, asociaciones e instituciones que colaboren en el buen desempeño de las labores. Además, considerar la contextualización del centro educativo, tomando en consideración las debilidades y las fortalezas que presenta el mismo para lograr adaptarse al contexto y de esta forma determinar cuáles son los objetivos más importantes por cumplir. Algunos de los objetivos o metas se encuentran intrínsecos en los proyectos que se promuevan en el centro educativo, sobre todo para mejorar estructura, relaciones interpersonales 0 referentes al currículo.

Para lograr verificar si se han logrado las metas propuestas es preciso evaluar el proceso y determinar la importancia de cumplir con lo acordado; de acuerdo con los sistemas de evaluación Lafourcade (1995, p.7) menciona que éstos "representan un esfuerzo conjunto de los miembros de la comunidad educativa por realizar un examen crítico de los propósitos, capacidades de logro, financiamiento, costos e impactos de los ámbitos de influencia del quehacer institucional". Solamente a través de criterios de evaluación, el coordinador logra en la institución educativa llevar un control de cómo está realizando su labor; tomando en cuenta que no sería una evaluación individual sino institucional.

Después de las consideraciones anteriores, en la actualidad y como lo establece Guadamuz (1979, p.42) "La coordinación requiere de una visión integradora, por parte de quien ejerza la coordinación"; por lo que los gestores deben ser personas abiertas a los cambios cotidianos de un mundo globalizado, es imposible seguir administrando como se hacía en años anteriores. Se hace necesario prestar especial atención a las exigencias de las sociedades para tratar de una u otra forma brindar una respuesta.

\section{Planificación.}

El Sistema Educativo del país debe responder a las exigencias de la sociedad, y también debe afrontar los cambios que sean prioritarios, aunque cumplirlo represente un gran desafío. 
Es por eso que se hace necesario conocer la realidad de la educación y la del modelo que se desea formar, esto es posible lograrlo si se realiza una adecuada planificación de la educación.

La planificación permite ver hacia el futuro tomando en cuenta decisiones planteadas en el presente. Para Venegas (2004) la planificación se define como:

Un proceso sistémico (sustentado en la teoría de sistemas, de tal manera que todos los elementos que intervienen interactúan entre sí y dependen unos de otros), integral, racional y constante en la previsión, organización y uso de recursos, todo con el propósito de tener mayores probabilidades de obtener los resultados deseados en un período determinado, según los objetivos previamente establecidos como parámetros. (p. 98)

La planificación educativa se considera un proceso continuo de construcción colectiva; en el cual participan y se involucran todas las personas que interactúan y hacen vida en la institución como lo son: los miembros de la Comunidad Educativa (el director (a), los docentes, los y las estudiantes, el personal administrativo, los y las conserjes, los guardas, las cocineras, los miembros de la comunidad) para determinar los fines de la institución y su concreción pedagógica, sobre la base del análisis de los documentos legales que orientan las políticas educativas. Además de planificar, estos deben realizar revisiones periódicas cada cierto tiempo.

Según Ander-Egg indica (1996):

Planificar es un proceso, es decir, es una actividad continua y unitaria que no termina con la formulación de un plan determinado, sino que implica un reajuste permanente entre medios, actividades y fines $y$, sobre todo, de caminos y procedimientos a través de los cuales se pretende incidir sobre algunos aspectos de la realidad. (p. 5)

Todo ello, con el propósito de organizar, diseñar, implementar, direccionar, coordinar, evaluar y sistematizar acciones y actividades que permitan el desarrollo del proceso de aprendizaje garantizando la transformación y la apertura de la comunidad en el quehacer de la institución, al proyectar su acción social y pedagógica en el proceso de formación de ciudadanía. 
El desarrollo de planificación de todo Centro Educativo debe sustentarse en los principios filosóficos que lo orientan y en los objetivos institucionales. Entre otras cosas, todo plan debe considerar los recursos presupuestarios, didácticos y humanos, debe ser creado para alcanzar unas metas que estén claramente definidas.

\section{Organización.}

La organización tiene incidencia en la calidad del quehacer educativo por diversos caminos, sobre todo en cuanto a la toma de decisiones de los responsables, por cuanto Santos (2001, p. 44) refiere que "la organización puede ser un factor propulsor de la calidad y un arma revolucionaria en la consecución de los cambios que la hagan posible". Lo anterior hace hincapié en la necesidad de tomar acuerdos conjuntamente, puesto que los miembros de las organizaciones no se preocupan únicamente por la satisfacción de sus metas, se preocupan también por los procesos mediante los cuales se toman acuerdos.

En ambos casos el bienestar individual va ligado a las operaciones de grupo y de organización.

Asimismo, Koontz et al. (2008) se refieren a la organización de la siguiente manera:

Es la agrupación de actividades necesarias para lograr objetivos, la asignación de cada grupo a un administrador con la autoridad necesaria para supervisarla, y la provisión de coordinación horizontal y vertical en la estructura de organización. Esta puede ser diseñada para clarificar quién debe hacer qué y quién es responsable de cuáles resultados. Debe ser diseñada para eliminar obstáculos al desempeño ocasionado por confusión e incertidumbre en las acciones para proporcionar redes de tomas de decisiones y de comunicaciones que reflejen y apoyen los objetivos. (p. 203)

En síntesis, se entiende por organización al proceso mediante el cual una persona o personas encargada(s) de distribuir funciones administrativas entre el personal asignado y de organizar la forma en que se llevan a cabo las acciones curriculares para el logro de los objetivos; a la vez informa a sus subalternos de los cambios que se presenten y es el encargado de tomar decisiones en la entidad. 
Para Fayol citado por Chiavenato (2006), la organización se refiere solo a la definición de la estructura y la forma de la administración; en consecuencia es estática y limitada.

Considera que la organización presenta dos significados:

1. Organización como ente social, en el cual las personas interactúan para alcanzar objetivos específicos. En esta acepción, la palabra organización indica cualquier iniciativa humana intencional, emprendida para alcanzar determinados objetivos. Las empresas constituyen un ejemplo de organización social.

2. Organización como función administrativa y parte del proceso administrativo (planeación, dirección, coordinación y control). En este sentido, organización significa el acto de organizar, estructurar y destinar los órganos encargados de la administración y fijar sus atribuciones e interrelaciones. (p. 71)

Resulta oportuno que ambos significados de organización son aplicables para los centros educativos, pero hay que hacer notar la función administrativa que cumple la organización desde el punto de vista de la gestión en las atribuciones e interrelaciones.

En síntesis, la organización es una función administrativa que sigue a la planificación. Determina y agrupa las actividades necesarias para conseguir los objetivos y las asigna a los cargos y personas respectivas, para que posteriormente sean ejecutadas.

\section{Ejecución.}

La ejecución como la planificación son los elementos más importantes para lograr el éxito de cualquier emprendimiento, el alcance de los objetivos y las metas dependerá mucho de estos dos procesos.

La palabra ejecución significa acción y efecto de poner en obra algo hasta completarlo, y comienza en el momento en que se ponen en marcha las acciones asignadas en la planificación (documento que ha sido aprobado). Para realizar todas las acciones es necesario contar con el equipo humano y medios materiales previstos para el diseño, por lo tanto, convergen la eficacia de los recursos humanos, didácticos y de infraestructura. En este mismo sentido Monterrey (2001) expone: 
En la fase de ejecución se realizan las diferentes tareas o actividades planificadas para obtener los resultados que lleven al logro de los objetivos. La ejecución es el núcleo de la gestión de proyectos, es la fase en la que se transforman los recursos en resultados esperados. El objetivo de la ejecución del proyecto es lograr su propósito. (p. 56)

Por tanto, la acción ejecutora enlaza y transforma lo deseado en el plan, en las tareas concretas que se han planificado. Para Monterrey (2001), la ejecución del proyecto debe comprender dos acciones básicas:

- La gestión y control de todos los recursos (humanos, financieros y físicos) de forma tal que se aseguren que los resultados del proyecto se obtengan de manera oportuna y eficaz en función de los costos.

- Seguimiento para asegurar que los resultados producidos sean los mismos que se identificaron en la fase de diseño.

- Asimismo, las funciones principales de la ejecución de proyecto son:

- Realizar las actividades y las tareas planificadas.

- Vigilar el progreso comparándolo con la planificación. (dirección)

- Hacer los ajustes necesarios en correspondencia con los problemas observados. (control)

- Mantener informada a la alta gerencia de los progresos y dificultades (comunicación). (p. 57)

Es claro que los proyectos exitosos dependen tanto de una buena planificación como de una ejecución eficaz, la cual va a depender de los factores anteriormente mencionados. Para llevar a cabo una buena ejecución debe velar para que las competencias profesionales de los miembros del equipo sean adecuadas a las tareas y actividades que tengan que desarrollar (manual de puestos establecidos), además entre ellas se tiene que dar las condiciones para que funcionen como un equipo de trabajo, por esto, debe dar prioridad a:

- Clasificación de funciones y responsabilidades de cada uno de los miembros 
- Comunicación abierta y espontánea

- Confianza y apoyo mutuo

- Habilidades intelectuales y sociales

Dentro de los equipos de trabajo la figura del responsable del proyecto es de suma relevancia al convertirse en el gestor del recurso humano y de los materiales, y si bien esa gestión ha de estar basada en la planificación previa a menudo surgirán imprevistos que lo obligarán a tomar decisiones rápidas, más o menos arriesgadas, con base en la evolución del propio proyecto.

En síntesis, la ejecución de proyectos es una tarea primordial en la conclusión de los objetivos, una buena planificación y una buena ejecución serán insumos transcendentales para el éxito de toda gestión dentro de una organización.

\section{Evaluación.}

La gestión de los proyectos de Educación Abierta para Jóvenes y Adultos debe comprender un ciclo articulado y progresivo de elementos de la gestión desde el momento mismo que se concibe la idea, este ciclo debe involucrar elementos como, coordinación planificación, organización, ejecución sin olvidar la evaluación, la cual debe ser vista, según D’Agostino (1995):

(...) un proceso dirigido a comprobar el grado de eficiencia y calidad de todos los elementos que convergen en la realización del hecho educativo, para valorar dicho grado frente a parámetros de referencia y decidir qué hacer respecto de este. (p. 14)

Es por ello que se encargará de valorar si el conjunto de actividades, medios utilizados y resultados obtenidos han cumplido los objetivos propuestos. En el caso de la presente investigación la evaluación de la oferta educativa del III Ciclo de Educación General Básica Abierta para Jóvenes y Adultos permitirá dar cuenta si todo el proceso llevado a cabo en la implementación de la modalidad ha cumplido con lo previsto en sus objetivos. 
Sin duda la cuestión central para realizar una evaluación siguiendo lo que formula FOREM (2000, p.34) en la definición de los objetivos, "solo podremos afirmar que los resultados obtenidos son los esperados o no, y en qué grado, si los objetivos han sido previamente definidos con precisión". Por lo tanto, para una adecuada evaluación se deben cumplir todas las otras etapas del proceso de gestión, primordialmente la de planificación que es donde se establecen los objetivos o metas a realizar.

Por su parte, Bhola (1992, p.9) se refiere al concepto de evaluación como "el proceso de juzgar el mérito o valor de algo"; por tanto, evaluar connota una carga axiológica importante y los juicios de valor no se pueden obviar. Este mismo autor considera que la evaluación debe organizarse razonablemente buscando la precisión como sea posible para garantizar que los resultados obtenidos sean públicamente defendibles, así mismo, se puede utilizar los datos que suministra la evaluación para generar información que pueda usarse en la planificación y la puesta en práctica de programas para mejorarlos.

Es necesario no encasillar la evaluación en la etapa final de un proyecto en torno al cumplimiento de los objetivos (evaluación de resultados) se debe ir más allá de las concepciones tradicionales ya que puede generar una visión algo limitada, existe amplia literatura que concibe la evaluación superando la simple valoración de resultados, la cual emplea tres niveles: una evaluación antes, durante y después del proyecto, en otras palabras una evaluación constante, transversal en toda organización.

De acuerdo con lo anterior, González (2005, p.21) define: "La evaluación como una acción transversal al ciclo de vida de los proyectos y programas de desarrollo". Es decir, no se debe esperar al final para realizarla, debe ser paralela a las etapas del proceso. La tabla 1 muestra a modo de resumen las principales etapas del ciclo de vida de la gestión así como los tipos de evaluación según objeto y temporalidad.

Tabla 1

Etapas de ciclo de vida de la Gestión

\begin{tabular}{|c|c|c|}
\hline Etapa & Fases & $\begin{array}{c}\text { Tipo de Evaluación } \\
\text { (según objeto y temporalidad) }\end{array}$ \\
\hline $\begin{array}{c}\text { Coordinación- } \\
\text { Planificación } \\
\text { (Antes del proyecto) }\end{array}$ & $\begin{array}{l}\text { Identificación } \\
\text { Planificación } \\
\text { Formulación }\end{array}$ & $\begin{array}{c}\text { Evaluación de } \\
\text { necesidades y } \\
\text { potencialidades } \\
\text { (Diagnóstico) } \\
\text { Evaluación previa }\end{array}$ \\
\hline
\end{tabular}




\begin{tabular}{|c|c|c|c|}
\hline Etapa & Fases & \multicolumn{2}{|c|}{$\begin{array}{c}\text { Tipo de Evaluación } \\
\text { (según objeto y temporalidad) }\end{array}$} \\
\hline & & $\begin{array}{l}\text { (valoración de } \\
\text { donantes) }\end{array}$ & \\
\hline $\begin{array}{l}\text { Organización-ejecución } \\
\text { (Durante el proyecto) }\end{array}$ & $\begin{array}{c}\text { Ejecución } \\
\text { Seguimiento }\end{array}$ & $\begin{array}{l}\text { Evaluación de Progreso } \\
\text { Evaluación de Proceso } \\
\text { Evaluación de fin de } \\
\text { proyecto }\end{array}$ & $\begin{array}{l}\text { Evaluación } \\
\text { Intermedia }\end{array}$ \\
\hline $\begin{array}{c}\text { Evaluación } \\
\text { (Después del Proyecto) }\end{array}$ & $\begin{array}{l}\text { Evaluación } \\
\text { Posterior }\end{array}$ & $\begin{array}{l}\text { Evaluación de } \\
\text { resultados e impacto } \\
\text { Evaluación de } \\
\text { experiencias o } \\
\text { Sistematización }\end{array}$ & Evaluación Ex post \\
\hline
\end{tabular}

Fuente: (González 2005, p. 15).

La acción de evaluar (valorar algo), se inicia desde los primeros momentos de concepción e identificación del proyecto, cuando se realiza la valoración de las necesidades y potencialidades de la población beneficiaria (diagnóstico). Es lo que se conoce como evaluación ex ante o evaluación previa. Además, durante la implementación del proyecto, es decir, en su ejecución a lo largo de un período más o menos largo, es necesaria la valoración continua del progreso de las actividades, del cumplimiento de planes iniciales, y del presupuesto. A esto se le llama evaluación del progreso de la intervención, o evaluación intermedia. Por último, la evaluación ex post o posterior que se realiza una vez terminado el proyecto o al cabo de un tiempo de finalizado. Esta valoración, la más completa de todo el proceso, toma prestado todos los conocimientos, avances e informaciones de las anteriores y será la que determine en última instancia si el proyecto fue exitoso en el logro de sus objetivos.

Los estudios evaluativos típicos (evaluación ex post) a gran escala demoran demasiado para que quienes tomen las decisiones esperen por los resultados. Las decisiones del programa con frecuencia dependerán de que se tome el pulso rápidamente acerca del estado del programa, como respuesta a esa necesidad se establecen las evaluaciones en micro, las cuales abarcan las instancias inferiores del sistema educativo como son las instituciones.

La evaluación como paso importante en el ciclo de vida de toda organización es un proceso sistemático con distintos matices y definiciones, sin embargo, uno de los aspectos primordiales de la misma es generar y divulgar información que genere decisiones oportunas 
tomadas de manera conjunta que mejoren el funcionamiento y calidad de la organización, así lo afirma González (2005):

Una herramienta que favorece la conciencia crítica y el aprendizaje en pro del mejoramiento de la calidad de nuestras instituciones, pero que requiere de un esfuerzo humano, técnico y económico. Se debe velar por una evaluación integral que no solo persiga el análisis descriptivo de resultados, sino también debe brindar información suficiente para las futuras toma de decisiones. (p. 15)

En conclusión se puede decir que existen modelos de evaluación diferentes debido a que los distintos especialistas han pasado por experiencias diferentes al aprender, hacer evaluación y al reflexionar sobre sus experiencias han utilizado diferentes puntos de vista y valores.

Contemplando los principales componentes de la gestión (ver Figura 1) coordinación, planificación, organización, ejecución y evaluación es preciso ubicarlos dentro del contexto de la investigación, siendo la gestión que desarrolla la modalidad de Educación Abierta para Jóvenes y Adultos. En este sentido es necesaria una fundamentación teórica que permita comprender la dinámica que se desarrolla cuando se trabaja con esta población.

Figura 1

Esquema del ciclo de la gestión

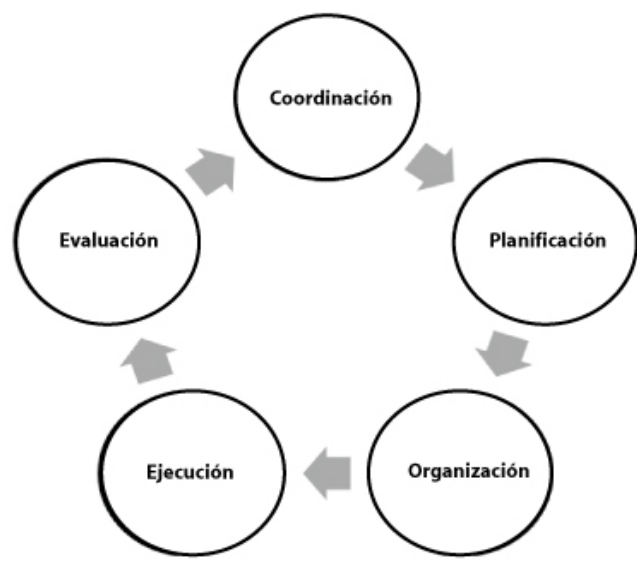

Fuente: Elaboración propia (2011). 


\section{Educación y gestión de Adultos (Andragogía).}

La educación es un proceso esencial en la vida de los seres humanos, en algunos casos este proceso se interrumpe postergándolo a la etapa de la adultez; lo cual genera que esta educación posea una serie de características que responda a las necesidades de esta población. Solano (1995) define el término andragogía como:

Ciencia de la educación de adultos, es un proceso dinámico, activo, real, concreto y fundamental práctico; por lo tanto, el proceso andragógico no consiste en depositar conocimiento, sino en desarrollar el pensamiento y la creatividad del adulto a través de metodologías participativas que permitan el logro de los objetivos planteados. (p. 9)

Después de realizar una definición de este término, hay que enumerar una serie de aspectos que justifiquen la importancia de la andragogía en la educación, y más aún entender los muchos matices que esta conlleva, entre algunos, tomar en cuenta el establecer un currículo que satisfaga a los actores que asistan a la oferta educativa de educación abierta.

Cabría preguntarse entonces, por qué se hace necesario la implementación de la educación para la persona adulta, qué aspectos habrían de considerarse, como lo expresa Cass (1974):

La educación básica para adultos ofrece a los hombres y mujeres de todas las edades la oportunidad de obtener las habilidades para actuar por sí solos con muy poca ayuda de los demás. Proporciona una secuencia de capacitación orientada hacia las necesidades de los adultos que carecen de nociones para leer, escribir y hacer cuentas, así como de los conocimientos necesarios para asegurarse una adecuada remuneración para el diario vivir. (p. 9)

Es importante ubicar en este particular, que la persona adulta se diferencia del niño y del joven en cómo se enseña, es una tarea específica a tomar en cuenta para la elaboración de un currículo distinto, pues se basa, según el caso, en la disparidad de los objetivos educativos, en especificidad de las características psicológicas de los adultos y en las situaciones vividas 
por estos últimos. A la hora de educar adultos se debe partir de la elaboración de un programa o currículo diferenciado, en el cual se tomen en cuenta dichas particularidades.

Existe un panorama amplio donde la educación de adultos se sustenta desde una base psicológica y con una visión global del hombre, donde el adulto se integra a un subsistema de una manera integral y con equidad.

Al igual que las diversas modalidades educativas, la educación de adultos necesita un adecuado proceso de gestión, en el cual se sistematice las principales actividades a realizar, por lo tanto, encontrar el camino para que esta se gestione apropiadamente, tendrá sustento dentro de la administración educativa y de las múltiples experiencias que, realizadas en otros países, sirven de ejemplo para el desarrollo de esta temática.

La educación de adultos contribuirá a la creación de una ciudadanía consciente y tolerante, al desarrollo económico y social, a la promoción de la alfabetización, la mitigación de la pobreza y la preservación del medio ambiente. Es una oportunidad, donde las personas pueden acceder al sistema educativo cuando lo requieran; por lo cual, el Estado debe proporcionar la oferta educativa para suplir las necesidades educativas de esta población.

\section{Referente metodológico}

La investigación se basó en un enfoque cualitativo de alcance exploratorio-evaluativo de manera que se examina un problema de investigación poco estudiado para el cual se tiene muchas dudas (Hernández, Fernández y Baptista, 2010).

Al ser la gestión de la modalidad de Educación Abierta un tema poco abordado tanto a nivel nacional como internacional, según indagaciones realizadas para la construcción del estado del arte el alcance exploratorio alcanza relevancia. También es un estudio descriptivo, al buscar como lo alude Hernández et al. (2010), al indicar que se buscan especificar las

propiedades, características y perfiles de las personas participantes del estudio o el fenómeno en sí, por lo que se pretende medir o recoger la información en relación con las categorías de análisis que orientan el estudio.

Participaron en la investigación la Asesora Nacional de Educación de personas jóvenes y adultas, el Director Regional de Educación de Occidente, la Asesora Regional de Educación 
de jóvenes y adultos, los Supervisores de cada Circuito (8), los Coordinadores Zonales (1), Coordinadores de Sede (5) y los profesores-docentes (32).

En cuanto a los instrumentos se ejecutaron entrevistas semiestructuradas a la Asesora Nacional de Educación de Personas Jóvenes y Adultos y al Director Regional de Educación de Occidente, también se aplicaron cuestionarios a coordinadores, supervisores de circuito escolar y docentes inmersos en el ámbito de la oferta educativa de educación abierta. Cada uno de los instrumentos busca responder a cada uno de los objetivos del estudio.

Para llevar a cabo el estudio se plantearon los siguientes objetivos:

\section{Objetivo general.}

Analizar la gestión de la oferta educativa del III Ciclo de la Educación General Básica Abierta para Jóvenes y Adultos en la Dirección Regional de Educación de Occidente.

\section{Objetivos específicos.}

1. Describir las funciones de la coordinación en las sedes y los proyectos de la oferta educativa del III Ciclo de la Educación General Básica Abierta para Jóvenes y Adultos

2. Determinar la planificación que implementa la oferta educativa del III Ciclo de la Educación General Básica Abierta para Jóvenes y Adultos en las sedes y en los proyectos

3. Reconocer la organización que se emplea en las sedes y en los proyectos de la oferta educativa del III Ciclo de la Educación General Básica Abierta para Jóvenes y Adultos

4. Identificar los procedimientos administrativos para la ejecución de los proyectos y sedes de la oferta educativa del III Ciclo de la Educación General Básica Abierta para Jóvenes y Adultos

5. Describir las actividades evaluativas aplicadas a los proyectos y sedes de la oferta educativa del III Ciclo de la Educación General Básica Abierta para Jóvenes y Adultos

Las categorías de análisis que orientaron el estudio según sus objetivos se indican en la siguiente tabla: 
Tabla 2

Categorías de análisis de la investigación

\begin{tabular}{|c|c|}
\hline Coordinación & $\begin{array}{l}\text { "El establecimiento y mantenimiento de la armonía entre las actividades } \\
\text { de los subsistemas de una dependencia o institución". Lépiz (1987, p. 88) }\end{array}$ \\
\hline Planificación & $\begin{array}{l}\text { "Proceso Sistémico integral, racional, y constante en la previsión, organización } \\
\text { y uso de recursos, todo con el propósito de tener mayores probabilidades de } \\
\text { obtener los resultados deseados en un periodo determinado, según los } \\
\text { objetivos previamente establecidos como parámetros". Venegas (2004, p. 98) }\end{array}$ \\
\hline Organización & $\begin{array}{l}\text { "Es la agrupación de actividades necesarias para lograr objetivos, la } \\
\text { asignación de cada grupo a un administrador con la autoridad necesaria para } \\
\text { supervisarla, y la provisión de coordinación horizontal y vertical en la estructura } \\
\text { de organización. Esta puede ser diseñada para clarificar quién debe hacer qué } \\
\text { y quién es responsable de cuáles resultados. Debe ser diseñada para eliminar } \\
\text { obstáculos al desempeño ocasionado por confusión e incertidumbre en las } \\
\text { acciones para proporcionar redes de tomas de decisiones y de } \\
\text { comunicaciones que reflejen y apoyen los objetivos". Koontz, Weihrich y } \\
\text { Cannice }(2008, \text { p. 203) }\end{array}$ \\
\hline Ejecución & $\begin{array}{l}\text { Fase en donde se realizan las diferentes tareas o actividades planificadas para } \\
\text { obtener los resultados que lleven al logro de los objetivos". Monterrey (2001, p. } \\
56) \text {. }\end{array}$ \\
\hline Evaluación & $\begin{array}{l}\text { Es un proceso dirigido a comprobar el grado de eficacia y calidad de todos los } \\
\text { elementos que convergen en la realización del hecho educativo, para valorar } \\
\text { dicho grado frente a parámetros de referencia y decidir qué hacer respecto de } \\
\text { este". D’Agostino (1995, p. 14). }\end{array}$ \\
\hline
\end{tabular}

Fuente: Elaboración propia (2012)

\section{Resultados}

A partir de la investigación realizada desde la gestión, para Guadamuz (1979, p.42) es notorio que "la coordinación requiere de una visión integradora, generalista, de un conocimiento globalista de parte de quien ejerza la coordinación". Al ser el proceso de coordinación una de las actividades más importantes de la gestión en los sistemas educativos y de acuerdo con las opiniones brindadas, es necesario que se integren diferentes aspectos relacionados con los cambios presentes en el mundo contemporáneo; por ello, se infiere que para que se dé una buena coordinación es indispensable la buena comunicación entre las distintas instancias, 
utilizando medios adecuados y oportunos. Además, integrar los esfuerzos por parte de los diferentes actores de la modalidad para lograr el cumplimiento de los objetivos propuestos.

Relacionado con los métodos de trabajo que presenta la oferta educativa estudiada no existe una unidad de acción que los armonice, de igual manera los criterios de evaluación presentan diferencias en lo que respecta a niveles macro (MEP) y micro (coordinadores), pues los primeros insisten en la importancia de las visitas para el monitoreo del trabajo de los diferentes funcionarios; a pesar de que esto es indispensable para un buen manejo del sistema; no se está ejecutando como debería.

Otros de los aspectos indispensables que fueron analizados en lo que respecta a la coordinación desde la gestión, es lo concerniente al currículo y la inducción al planeamiento; de los cuales se obtuvo una posición favorable ya que, según las respuestas obtenidas sí se habilitan los espacios para la modificación del currículo y por ende para la capacitación del planeamiento como tal. Aunado a esto se encuentra la implementación del FODA (Fortalezas, Oportunidades, Debilidades y Amenazas) como instrumento para mejorar la modalidad, y tomando en cuenta las necesidades que presenta la población y el contexto en el cual se encuentran inmersas las instituciones.

En lo concerniente a la publicación de las pruebas, la calendarización de actividades y controles de asistencia de los estudiantes, se realiza una buena coordinación desde oficinas centrales, ya que estas se encuentran establecidas con antelación y permiten el buen cumplimiento de los objetivos y por ende el buen funcionamiento de la modalidad.

Existen una serie de factores que pueden aportar desde la administración de la educación, específicamente desde el sistema de coordinación, para mejorar la calidad de la oferta educativa, entre estos: una adecuada comunicación del Director Regional, Asesora Nacional de Educación para Adultos, Asesora Regional de Educación para Jóvenes y Adultos, Supervisores Educativos, Coordinadores de sede y docentes, que favorezca el proceso de gestión. También, se debe contar con adecuados métodos de trabajo para el buen desempeño de la modalidad, que a través de recursos y estrategias, logren responder a los imprevistos que se presenten y consideren las características propias del contexto en el cual se encuentre inmersa la oferta educativa.

El sistema de coordinación como génesis del ciclo de la gestión debe desarrollarse de forma óptima, para que las siguientes etapas puedan construirse sobre esta base, lo que 
incidirá en una educación que genere una mayor formación y equidad entre los educandos que asisten a esta oferta en comparación con los que acuden a otros sistemas formales, fortaleciendo de esta manera el nivel educativo del país.

A nivel de las instancias del MEP existe una planificación que permite la implementación de la modalidad de una manera sistemática; sin embargo, retomando que la modalidad se divide en sedes y proyectos, es visible que esta se hace más expedita en los centros donde sí existe un coordinador, quien como gestor, es el encargado de llevar a cabo las funciones de dicho proceso, tal como lo apunta Esquivel (2009, p. 42): "El administrador de la educación se convierte en un gestor que debe desarrollar estrategias de calidad en la institución".

Del planteamiento anterior, y con respecto de los datos analizados, se puede afirmar que la figura del coordinador es imprescindible para buscar los caminos y procedimientos adecuados en el cumplimiento de los objetivos propuestos dentro de esta oferta educativa, integrando la confección de los planes con la asignación de los recursos humanos, didácticos y financieros.

En relación con la organización de la modalidad en las sedes y los proyectos, los datos reflejados confirman que la organización es una de las funciones primordiales de la gestión de los programas de Educación Abierta para Jóvenes y Adultos; en este sentido se muestra cómo esta viene estructurada desde las instancias superiores del MEP, lo cual incluye una adecuada articulación entre ellas, facilitando la distribución de funciones y los medios de comunicación oportunos para el máximo desempeño de la modalidad. Por tanto, la administración de la educación, como medio para garantizar la eficacia y eficiencia de esta modalidad, viene a cobrar un papel preponderante, como lo afirma Chiavenato (2006):

La tarea de la administración pasó a ser la de interpretar los objetivos propuestos por la organización y transformarlos en acción organizacional a través de la planeación, la organización, la dirección y el control de todos los esfuerzos realizados en todas las áreas y en todos los niveles de la organización, con el fin de alcanzar tales objetivos, de la manera más adecuada a la situación y garantizar la competitividad [...]. (p. 10)

Es así como las acciones organizativas comprenden una etapa fundamental en el proceso de la gestión de las sedes y los proyectos, al ser estos los receptores de las acciones 
planificadas, son los que deben efectuar una adecuada organización y mantenerla a través del periodo escolar.

Para Monterrey (2001), las funciones principales de la ejecución de proyectos son:

- Realizar las actividades y las tareas planificadas.

- Vigilar el progreso comparándolo con la planificación. (dirección)

- Hacer los ajustes necesarios en correspondencia con los problemas observados (control). (p. 57)

La ejecución es una de los componentes necesarios de la gestión para comprobar que las actividades planificadas se cumplan adecuadamente, viendo esta como un mecanismo de control y fiscalización de las acciones.

Dentro del ciclo de la gestión que se desarrolla en la modalidad del III ciclo de la Educación General Básica Abierta para Jóvenes y Adultos, la ejecución es una de las fases que devela que la coordinación, planificación y organización son efectivas si a la hora de ponerlas en práctica se logra desarrollar los propósitos y objetivos para los cuales se creó este programa, además articula los recursos humanos, didácticos y financieros para una gestión eficaz y realizar los ajustes pertinentes en caso de que algunos de estos elementos presente alguna debilidad.

Desde el punto de vista de la administración de la educación, la evaluación logra generar nuevas acciones en beneficio del funcionamiento de la oferta educativa del III Ciclo de la Educación General Básica Abierta para Jóvenes y Adultos ya que la información que se genera, a través de este proceso, faculta estrategias que permiten mantener una constante retroalimentación de todo el ciclo de la gestión para iniciar uno nuevo.

A manera de síntesis, como se menciona a través del estudio, el ciclo de la gestión contempla cinco elementos que han sido analizados a la luz de la oferta educativa del III Ciclo de la Educación General Básica Abierta para Jóvenes y Adultos.

Es así, como desde el sistema de coordinación, para mejorar la calidad de la oferta educativa, se necesita: una adecuada comunicación del Director Regional, Asesora Nacional de Educación para Adultos, Asesora Regional de Educación para Jóvenes y Adultos, Supervisores Educativos, Coordinadores de sede y docentes, que favorezca el proceso de gestión. Además 
de contar con adecuados métodos de trabajo para el buen desempeño de la modalidad que, a través de recursos y estrategias, logren responder a los imprevistos que se presenten y consideren las características propias del contexto en el cual se encuentra inmersa la oferta educativa.

El sistema de coordinación como génesis del ciclo de la gestión debe desarrollarse de forma óptima para que las siguientes etapas puedan construirse sobre esta base, lo que incidirá en una educación que genera una mayor formación y equidad entre los educandos que asisten a esta oferta en comparación con los que acuden a otros sistemas formales, fortaleciendo el nivel educativo del país.

En este mismo sentido, la segunda etapa de análisis, la planificación, se organiza desde el MEP en forma sistemática; sin embargo, retomando que la modalidad se divide en sedes y proyectos, es visible que esta se hace más expedita en los centros donde sí existe un coordinador quien, como gestor, es el encargado de llevar a cabo las funciones de dicho proceso.

Del planteamiento anterior y los datos analizados en esta categoría se puede afirmar que la figura del coordinador es imprescindible para buscar los caminos y procedimientos adecuados en el cumplimiento de los objetivos propuestos, dentro de esta oferta educativa, integrando la confección de los planes con la asignación de los recursos humanos, didácticos y financieros.

En referencia a la tercera etapa de la gestión los datos reflejados confirman que la organización es una de las funciones primordiales de la gestión en los programas de Educación Abierta para Jóvenes y Adultos.

Se muestra cómo viene estructurada desde las instancias superiores del MEP, lo cual incluye una adecuada articulación entre estas, facilitando la distribución de funciones y los medios de comunicación oportunos para el máximo desempeño de la modalidad. De esta manera, la ejecución como otro de los componentes de la gestión permite comprobar que las actividades planificadas se cumplan adecuadamente, lo que sirve de mecanismo de control y fiscalización de acciones, develando que la coordinación, planificación y organización sean efectivas a la hora de ponerlas en práctica para el logro de propósitos y objetivos para los cuales se creó este programa; además, articula los recursos humanos, didácticos y financieros 
para una gestión efectiva y realizar los ajustes pertinentes en caso de que algunos de estos elementos presenten debilidad.

Desde el punto de vista de la administración de la educación, la quinta etapa: evaluación, logra generar nuevas acciones en beneficio del funcionamiento de la modalidad, ya que la información que se genera, a través de este proceso, faculta estrategias que permitan mantener una constante retroalimentación de todo el ciclo de la gestión para iniciar así uno nuevo.

Es así como el desenvolvimiento y la articulación entre las cinco etapas garantiza, desde la teoría, procesos que facultan, desde la administración de la educación, el cumplimiento de los propósitos para los que fue creada la modalidad tal y como lo demuestra la siguiente figura:

Figura 2

Ciclo de Gestión propuesto para el mejoramiento de la administración del III Ciclo de la Educación General Básica Abierta para Jóvenes y Adultos del MEP

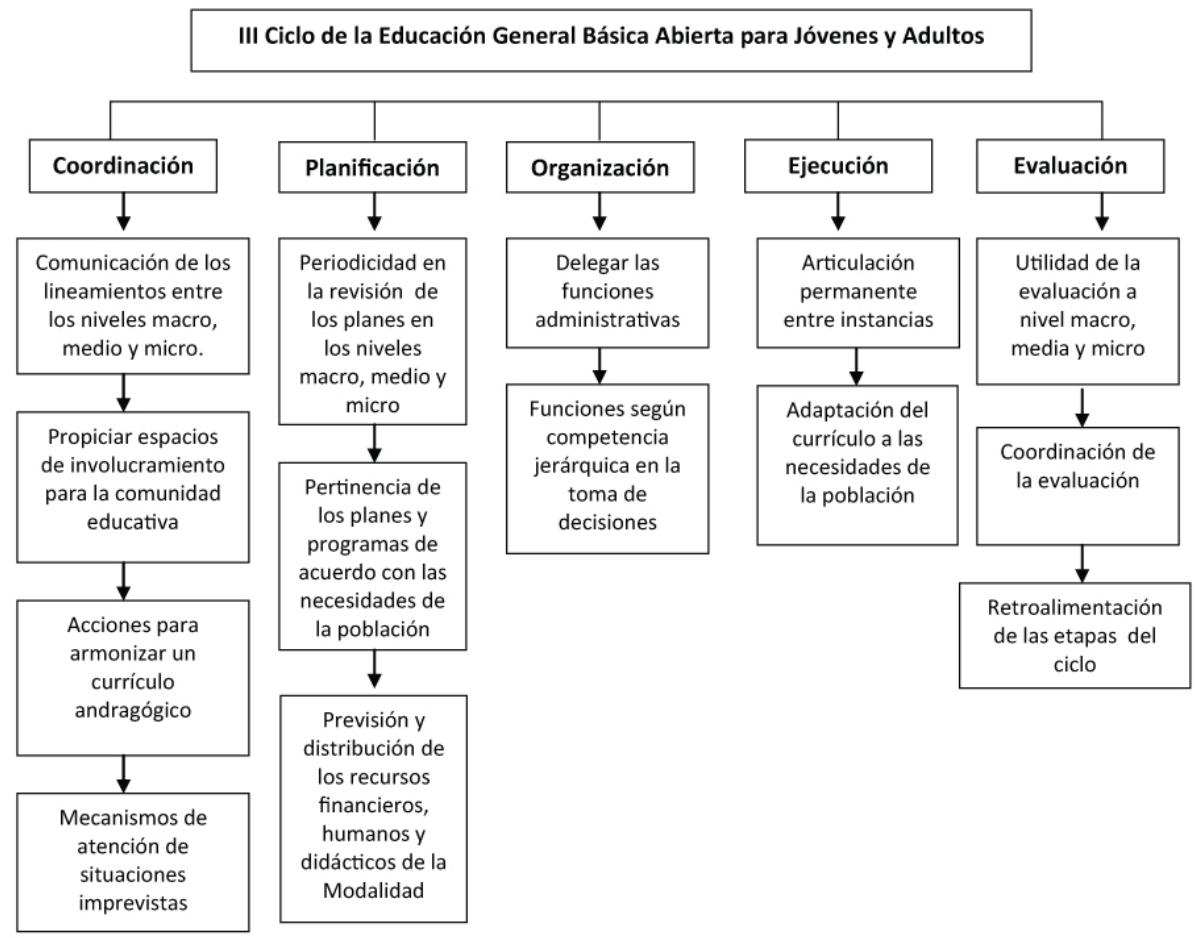

Fuente: Elaboración propia (2011). 


\section{Conclusiones}

Entre las principales conclusiones del estudio se sintetizan las siguientes:

- Es necesario establecer adecuados medios de comunicación, los cuales son indispensables para evitar la confusión, la incertidumbre y los conflictos provocados por la distorsión de la información, que son los responsables de provocar obstáculos que generan una atrofia comunicativa, entre los niveles medios y bajos.

- Visto desde la administración de la educación, se hace notar la necesidad de mantener apropiados métodos de trabajo en los programas de Educación Abierta, ya que esto permitirá desarrollar proyectos que mejoren su desempeño. Por lo cual se hace necesario que se ejecuten las acciones pertinentes (comunicación, esfuerzos involucrados, metodología de trabajo, comunicación de los lineamientos, involucrar participantes, currículo, planeamiento didáctico, atención de situaciones imprevistas, calendarización y procedimientos, sistema de evaluación, proyectos extracurriculares y asistencia) para que la coordinación sea óptima.

- La evaluación, desde la coordinación es deficitaria. Se demuestra que no se realiza en forma constante e integradora para garantizar la calidad del servicio que se brinde. Solamente, a través de criterios de evaluación (estandarizar la calidad de la evaluación, calendarización, utilidad de las estadísticas de las pruebas y unificación de criterios), la modalidad del III Ciclo de Educación General Básica Abierta para Jóvenes y Adultos, logrará llevar un control de cómo se están realizando las funciones, tomando en cuenta que no sería una evaluación individual sino institucional.

- Se hace notar, desde la administración de la educación, la necesidad de una apropiada coordinación de todas las partes, donde se establezcan formas de desarrollar el currículo, de acuerdo con las necesidades de la modalidad donde supervisores, coordinadores y docentes mantengan contacto para lograr dicho fin.

- Se denota escasez de espacios para vincular a la comunidad educativa en proyectos extracurriculares ni para coordinar metodologías de trabajo, reflejando que para los altos mandos del programa sí existe, pero a nivel micro no se dan, teniendo como resultado deficiencias en la coordinación. 
- Se evidencia deficiencia en el control de asistencia de los estudiantes, por parte de los docentes, esto influye directamente para la apertura de los cupos mínimos necesarios y la asignación presupuestaria por parte del MEP.

- El personal docente no tiene el conocimiento sobre los planes a nivel institucional, esto se contrapone a lo que desde la administración de la educación, debería ser una gestión articulada, responsable a través de un proceso de construcción colectiva, garante de que la planificación sea parte de todos los niveles de la organización.

- Se observa la necesidad de que la evaluación de la planificación sea un proceso constante, realizándolo de forma paralela a las etapas del proceso y no de forma aislada como se ha venido realizando con el programa nacional en periodos de 10 años, espacio muy amplio que abarca cambios en la sociedad y en las necesidades de las personas que optan por esta oferta educativa.

- La mayoría de los docentes del estudio no son parte de la revisión periódica del plan institucional. Esta situación se puede deber a una falta del sentido de pertenencia de los docentes a este sistema educativo, puesto que la mayor parte del personal trabaja con un recargo de funciones y se subestima al ser vista esta como una oferta educativa no formal.

- Los docentes son los mediadores del proceso educativo, estos detectan la deficiencia del Plan Nacional de dicha modalidad, pues no logra el enfoque andragógico adecuado a la población a la cual se dirige.

- Una situación resultante es la mezcla de formas de aprendizaje, puesto que, por un lado las diferencias de edad hacen que el currículo sea adaptado a los más jóvenes pero no a los de edad adulta. Por otro lado, si se adaptaran los programas a los adultos como tales, podría suceder que entonces se aleje de la realidad de los más jóvenes.

- A nivel de las instancias del MEP (macro, medio y micro) existe una planificación que permite la implementación de la modalidad de una manera sistemática; sin embargo, retomando que la modalidad se divide en sedes y proyectos, es visible que ésta se hace más expedita en los centros donde sí existe un coordinador quien como gestor, planifica las funciones de dicho proceso. 
- En la organización, a nivel macro, sí se presenta una adecuada articulación con lo referente a comunicación; sin embargo, a nivel micro, se presentan algunas deficiencias en este mismo aspecto, afecta de una u otra manera el funcionamiento del sistema.

- En los recursos a nivel de la ejecución, son los didácticos los que presentan mayor deficiencia, en comparación con los humanos y financieros. Hay que hacer notar que la articulación de los tres es indispensable para una optima ejecución de la modalidad del III Ciclo de la Educación General Básica para Jóvenes y Adultos.

- Al no existir pertinencia de los planes y programas en esta modalidad, el docente se ve obligado a adecuar el currículo para que responda a las necesidades de la población que atiende.

- El criterio de los supervisores y coordinadores se aleja de la realidad que expresan los docentes ya que esta parte del proceso de gestión, no es de su competencia ni tampoco se les comunican resultados o utilidad de las evaluaciones realizadas a nivel nacional.

- Analizando todos los componentes del ciclo de la gestión desarrollados en esta modalidad y desde la administración de la educación, en la etapa de evaluación se muestra gran debilidad, puesto que no existen estadísticas que reflejen la funcionalidad y efectividad de esta en el sistema educativo nacional.

- Al fortalecer esta modalidad de educación, el país promueve el cumplimiento de su legislación y de los convenios internacionales a los cuales se ha incorporado, incentivando la movilidad social y escolaridad de los ciudadanos.

- La administración de la educación se convierte en la ruta de cambio necesaria para una nueva forma de gestionar el sistema educativo nacional y sus distintas modalidades, convirtiéndose en un reto para los nuevos gestores, hacer posible la integración de todos los ámbitos que la afectan: el contexto, la comunidad educativa y la globalización.

\section{Referencias bibliográficas}

Ander-Egg, E. (1996). La planificación educativa. Argentina: Magisterio del Río de la Plata. Bhola, H.S. (1992). La evaluación de proyectos, programas, y campañas de "Alfabetización para el Desarrollo". Santiago: UNESCO.

Bolaños, G. y Molina, Z. (2008). Introducción al currículo. San José, Costa Rica: EUNED. Lafourcade, P. (1995). Evaluación institucional. San José, Costa Rica: EUNED. 
Cass, A. W. (1974). Educación básica para adultos: manual para educadores, formadores de docentes y directores de programas de educación básica para adulto. Buenos Aires: Troquel.

Casassus, J. (2000). Problemas de la gestión educativa en América Latina. Chile: UNESCO.

Castillero, A. (2003). Gestión y supervisión en el Centro de Educación Básica. Cartago, Costa Rica.

Chavarría J., E. y Araya P., A. (1984). Perfil de la administración educacional. Departamento de Investigación Educacional. San José, Costa Rica.

Chiavenato, I. (2006). Introducción a la teoría general de la administración. México: Mc Graw-Hill Interamericana.

D’Agostino, G. (1995). Aspectos teóricos de la evaluación educacional: una orientación para su puesta en práctica en la enseñanza primaria. San José, Costa Rica: EUNED.

Esquivel C., G. (2009). Análisis de la gestión directiva basada en competencias para la Escuela Quince de Agosto. Tesis sin publicar. Universidad de Costa Rica, Escuela de Administración Educativa.

FOREM. (2001). Calidad en la ejecución de proyectos de formación continua. Madrid: FOREM.

González G., L. (2005). La evaluación en la gestión de proyectos y programas de desarrollo: una propuesta integradora en agentes, modelos y herramientas. España: VitoriaGasteiz.

Guadamuz, L. (1979). La administración educativa y su relación con la administración pública. San José, Costa Rica: EUNED.

Hernández, R.; Fernández, C. y Baptista, P. (2010). Metodología de la investigación. México: Mc Graw Hill.

Koontz, H.; Weihrich, H. y Cannice, M. (2008). Administración: una perspectiva global y empresarial. México: Mc Graw Hill.

Ministerio de Educación Pública [MEP]. (2010). Directrices y lineamientos administrativos para las modalidades de educación de personas jóvenes y adultas. Oficio DDC 2612-2010 de 2010, Dirección de Desarrollo Curricular. San José, Costa Rica: Editorial MEP.

Monterrey G., P. (2001). Fundamentos de la gerencia de proyectos en nutrición. Cuba: Infanta.

Santos G., M. (2001). Enseñar o el oficio de aprender: organización escolar y desarrollo profesional. Argentina: Homo Sapiens Ediciones.

Solano S., O. (1995). Manual sobre estratégicas metodológicas del aprendizaje andragógico. San José, Costa Rica.

Terry G., R. (1980). Principios de administración. México: Continental.

Venegas J., P. (2004). Planificación educativa: bases metodológicas para el siglo XXI. San José, Costa Rica: EUNED. 